\title{
Asynchronous Network Discussions as Organizational Scaffold Learning: Threaded vs. Flat-Structured Discussion Boards
}

\author{
Chih-Hsiung Tu \\ Michael Blocher \\ Lawrence Gallagher \\ Northern Arizona University
}

\begin{abstract}
Threaded and flat-structured discussions to support online learning present online educators with new and challenging dilemmas. Both formats apply different interfaces, structures and require different organization techniques for presenting postings which may affect the ways students participate. Both formats may impact online learning differently in terms of the organizational scaffold of learning. The purpose of this study was to examine experiences of online students and compare the impact that threaded and flat-structure discussion boards exerted on learning. This study concluded that both formats critically enhanced and inhibited students' online discussions. Both formats may empower discussion participants differently and with strategic design, can engage learners in more meaningful, deeper and higher order of thinking. To argue that one is better or more effective than the other or to dispute that educators should seek the potential of applying one tool to replace the other is inappropriate. This study proposes that learners should shift their roles from online learners to "network" learners, while instructors should shift their instruction paradigms from online discussions to "network" discussions. Educators should empower network learners to define the organizational scaffolding of their network learning structures and environments. Effective network instructional strategies for network discussions are recommended.
\end{abstract}

Keywords: interface, flat-structured discussions, threaded discussions, Open Network Learning Environments (ONLE), network instructional strategies

\section{Introduction}

Online discussion board is considered one of the most effective instructional strategies to engage learners in knowledge construction and to teach critical thinking skills (Cheong \& Cheung, 2008; de Leng et. al., 2009). Educators continue to integrate asynchronous online discussions boards to enhance learning in completely online, hybrid, or Web- enhanced instruction because research has evidently concluded that online discussions support learners and instructors to challenge, reform, and synthesize their current views of knowledge through in-depth interaction with other learners (Garrison, Anderson, \& Archer, 2001). Researchers agree that asynchronous online discussions frame a constructivist learning approach to enhance interaction, analysis, and collaboration of 
discussion participants (Bonk \& Dennen, 2007) and critical thinking skills (Richardson \& Ice, 2010). More specifically, research has concluded that asynchronous threaded discussions effectively facilitate learners' meta-cognitive awareness and development of self-regulatory processes and strategies (Vonderwell, Liang, \& Alderman, 2007).

Online asynchronous discussion board is frequently conducted in a text-based format as threaded, tree, nested, or parent-child interface. Instructors or learners may initiate new threaded discussion topics and the other can reply to the original discussion topic. The original discussion topic and the reply messages are displayed in a threaded, tree, or nested format with various fields such as authors, topics, time stamps, etc. Often asynchronous online discussion boards are integrated with a Course Management System (CMS) or a Learning Management System (LMS) such as Blackboard or WebCT. These online discussion activities are generally required by the course instructors; therefore, learners are required to reply to discussion questions and/or others' postings within the whole class or in groups.

Since the advent of Web 2.0 tools, many educators integrate Web 2.0 discussion boards to support online discussions or to replace threaded discussion boards. In general, Web 2.0 discussion boards apply a "flatstructured" format to display the discussion postings chronologically with additional social network features such as tagging, RSS (Really Simple Syndication), widgets, tag clouds, social network linkages, etc. Flatstructured discussion board applies a simple interface; all postings are displayed in a single level, rather than in a threaded or nested reply structure. Flat-structured discussion formats can frequently be found in blogs, wikis, and social network sites (Twitter, Facebook, Ning).
Educators integrate Web 2.0's flatstructured discussions without knowing the different interface features because the technology is new to educators. These two different discussions formats harness different interfaces to exhibit online discussion postings. Educators have not examined how these different interfaces may affect online discussions. The purpose of this study was to investigate experiences of online students and to compare the impact of threaded and flatstructure discussion boards on learning.

\subsection{Flat-Structured Discussion \& Social Networking Environments}

In addition to a chronologic display interface, flat-structured discussions are normally equipped with optional social network features such as social tagging, tag clouds, RSS, widgets, social network linkages, and mobile learning linkages to allow discussion participants to access and to understand discussion content from different perspectives which are not available in a threaded format. These network technologies still require instructors to integrate these tools with the requirement that participants utilize them to support online discussions.

Flat-structured discussions generally allow participants to tag their postings in a field that is separated from the postings. The tags function as keywords. Participants can provide tags based on their posting's content or social context to enrich the postings. Some tools may feature a tag or word clouds to assist participants to view multiple tags based on the frequency of tags with larger fonts.

Really Simple Syndication (RSS) allows participants to subscribe to updates, or any new changes or new activity in subscribed discussions. Participants can apply RSS readers, such as Google Reader (http://reader.google. 
com), to organize multiple RSS subscriptions. This social network feature allows participants to remain updated on new postings without visiting the actual discussion pages.

Web 2.0 widget technologies allow participants to link flat-structured discussions to different Web pages, personal portals such as iGoogle (http://igoogle.com), or mobile Apps and devices. These social networking technologies allow participants to sign on, manage, and organize flat-structured discussions at their preferred locations and with the technologies at hand (e.g., mobile telephones).

Unlike threaded discussions, flat-structured discussions can visually display participants' names and profiles such as pictures, avatars, and links to personal profiles along with their postings. Participants can upload their own pictures or avatars, configure their personal profiles, or network to become friends, fans, or followers. Every time participants post postings, their pictures or avatars are attached to their postings along with links to their profiles. This feature provides a visual interface to support discussions with social and personal touches and effects. Normally, participants can click the pictures or avatars to view the authors' profile. Some flat-structured discussion boards are equipped with widgets to automatically feature community highlights such as hot topics, top discussion contributors, visual highlights of members, etc. These social network mechanisms are powered by the network servers and they display discussion activities based on real-time data from different perspectives.

Both threaded and flat-structured discussions integrate and utilize different interfaces. How they may impact discussion participants is unclear and has not been addressed by researchers and educators. Abetter understanding could be gained by researchers examining how these two discussion interfaces may impact online discussions.

\subsection{Learning Impacts}

Human thinking, knowledge presentations, and constructions may not be as simple as hierarchical forms. Human thinking may be symbolized as a more networking, weaving format (Educause, 2008). In other words, humans reflect and synthesize various types of ideas/viewpoints to construct a new set of knowledge. Hillman, Willis, \& Gunawardena (1994) emphasized the importance of learnerinterface interaction. Branching and replying cause threaded discussions to become off track, and following a thread that has branched can be discombobulating and unnatural, which commonly, forces participants to initiate a new thread if they want to return to the initial topic. Flat-structured discussions require participants to read all postings to promote meta-cognition and self-regulated skills to achieve higher learning.

\subsection{Weaving \& Synthesizing Postings}

Educators would agree synthesizing various posted ideas and viewpoints into one coherent position in online discussions would be more valuable than replying to one idea or viewpoint in a more limited sense. To achieve a synthesizing function in threaded discussion, online learners view the content of each posting to determine which postings require a response. Before online learners can synthesize content of a threaded interface they are forced to search by a clicking motion that may defuse their thinking. Typically, participants read several postings and select one that seems appropriate for response, inducing parent-child postings. Participants tend not to read every posting before they reply (Feldstein, 2005). Therefore, the approach 
of reading one and replying to one becomes common in threaded discussions.

\subsection{Engage Learners In Organizational Scaffolding}

Flat-structure discussion boards generally have other networking technologies to help support learning, such as RSS and social tagging, to achieve a better organizational scaffold. Flat-structured discussion boards are in their raw format, or chronological organizations. Chronological formats can present difficulties in comprehending discussion contents. Requiring learners to integrate RSS (Lee, Miller, \& Newnham, 2008) and social tagging (Godwin-Jones, 2006) as a way of organizing the discussion postings into their own organized structures is required. In other words, online learners define their own organizational learning scaffold rather than following the pre-determined hierarchal structures.

\subsection{Authentic Learner-Centered Learning}

Learners follow the hierarchal and threaded structures in threaded discussions to construct their knowledge through limited learner-centered learning while flat-structured discussions require learners to organize, manage, and regulate their own discussion learning structures by using other social and network mechanisms. This is a more authentic learner-centered experience and learners are empowered to shape learning technology.

Current literature indicates that there are several weaknesses in the threaded discussion format. Research has indicated that flatstructured discussion formats have the potential to resolve the weaknesses of threaded discussions. Educators should not overlook integrating a flat-structured format to improve online discussion instructions. Obtaining comprehensive understanding of students' learning experiences in both discussion formats is necessary. The purpose of this study was to investigate the experiences of online students and to compare the learning impact of threaded and flat-structure format discussions. The research question was: what differences do students experience when comparing between the flat-structured discussion format to threaded discussion format?

\section{Methods}

Forty subjects from two complete graduate courses (two different groups enrolled in two different sessions of the same course) taught by the same instructor participated in both flat-structured and threaded discussion formats for two weeks each respectively, using Blackboard Vista threaded discussions and Wetpaint wiki discussions in fall 2009. Participants were enrolled in the second year of a completely online master program in Educational Technology. This course was selected because it had more than one session and the students were familiar with threaded discussion format because they were in their second year of their master program. Twentytwo participants were female $(55 \%)$. The most predominant ethnic group was Caucasian $(\mathrm{n}=22,55 \%)$, and the Latino was the second largest one $(n=10,25 \%)$. The remainder of the participants were African American $(n=3,7.5 \%)$, Native Americans $(n=3,7.5 \%)$, Asians $(n=1,2.5 \%)$, and others $(n=1,2.5 \%)$. Both threaded discussion format and flatstructured format of online discussions were required and graded. Students participated in threaded discussions for the first two weeks and then participated in wiki's flat-structured discussions for another two weeks. Each discussion format contained 3-4 discussion topics related to course content, initiated by the instructor. Students were required to participate in both discussion boards regularly 
throughout four weeks of online discussions. Flat-structured discussion board had social tagging, RSS, and social networking features (profiles, personal pictures/avatars) available and participants were encouraged, but not required, to use these.

After four weeks of discussions, participants were asked to make their discussion reflections on their blogs and online discussions. Additionally, the data were collected through in-depth interviews. Eight randomly selected volunteers participated in individual interviews concerning their online discussion experiences with both discussion formats. This was a casual conversation conducted between the researcher and participants on Elluminate, an online conferencing system. The interviews were recorded with the participants' consent for the data analysis. Eight semi-structured indepth interviews were conducted with the participants to understand their experiences with both discussion formats. Examples questions asked include:

- How did flat-structured discussion impact your learning when compared to threaded discussions?

- What are your relationships with other online participants?

- What Web 2.0 technologies did you use to enhance and support the flatstructured discussions?

- How well did they perform?

\section{Data Analysis}

The data were collected and analyzed according to the qualitative method. Blog reflection data, online discussion postings, and semi-structured in-depth interview transcripts were collected for data analysis. Four dimensions from the Constructs of Web 2.0 Learning Environments ( $\mathrm{Tu}$,
Blocher, \& Roberts, 2008) were employed to guide participants' Blog reflections, online discussions, and semi-structured in-depth interviews on their discussion experiences in both discussion formats and to guide researchers in analyzing the data. Participants had a great deal of experience in using threaded discussion during their master degree process, and data collection and analyses were used to compare the participants' flat-structured discussion experiences to their threaded discussion experiences.

Two individuals coded the data independently utilizing the four dimensions from the Constructs of Web 2.0 Learning Environments ( $\mathrm{Tu}$, Blocher, \& Roberts, 2008): Cognitive, Social, Networking, and Integration. Orientation was provided to both coders and included: (a) explanations of the coding process; (b) written coding rules and guidelines; (c) examples and non-examples; and (d) practice with sample data. Both coders acquainted themselves with the particulars of the coding scheme and reached a mutual agreement about the coding category to be selected.

Initially, the four codes (cognitive, social, networking, and integration) were applied to code the data. The four dimensions were expanded into eight categories. Thinking and density of discussion context emerged from cognitive dimension; context-oriented discussion environment, and social network features emerged from social dimension; social tagging, and network mechanisms articulated network dimension; and collaborative effectiveness, and community sense attended the integration dimension.

Triangulation methods were utilized to achieve a better understanding about the participants' experiences in both threaded and flat-structured discussion formats, but not as a validation process. Data triangulation 
consisted of time, space, and personal triangulation. Method triangulation consisted of Blog reflections, online discussions, and semi-structured in-depth interviews.

\section{Results}

This study identified that both threaded and flat-structured discussion formats impacted learners' discussion experiences in thinking, density of discussion context, context-oriented discussion environments, social network features, social tagging, network mechanisms, collaborative effectiveness, and community sense.

$\mathrm{Tu}$, Blocher, and Roberts' (2008) Web 2.0 Learning Environment Constructs including four dimensions (Cognitive, Social, Networking, and Integration) that were applied to analyze participants' experiences in both discussion formats from blog reflections, online discussions, and semi-structured indepth interviews.

The Cognitive Dimension focuses on the process of the individual "thinking" about their engagement in and the culture within, "density of discussion context." Participants think about what they will contribute, and how and with whom they will contribute, which is then implemented as they construct their knowledge through online discussions. Social dimension refers to learners and their networking mediated relationships in "context-oriented discussion environments" with others and focuses both on the individual and the social contexts by integrating "social network features."

Networking dimension refers to the network technology architecture such as "social tagging" that empowers learners to select different "network mechanisms" to organize and manage their network learning environments through interoperable learning architectures. Integration dimension refers to the engagement of learners in "collaborative effectiveness" and "community sense" related activities via network social ritual.

\subsection{Cognitive Dimension}

Participants' experiences with different interfaces impact their cognitive learning process and were clustered as thinking, sense of discussion context, discussion posting skills, mental models, and learning perception shift. Participants indicated that both formats enhanced and inhibited their cognitive learning discussions.

\subsubsection{Thinking}

Both interfaces enhance and inhibit participants thinking processes. Participants reflected that flat-structured interfaces allowed ideas and viewpoints to be synthesized and they must think what they need to think; however, they indicated this process is extremely difficult to follow who is talking to whom about what and that inhibits thinking. Visually, flat-structured interface does not offer the relationships between and among related postings. With threaded interface, students indicated they can read one posting and reply to it easily and quickly (parent-child postings); however, they had to click and read many postings before they could synthesize all postings. By the time they were ready to synthesize their ideas, they could not remember the ideas to begin with because of the inability to organize the postings on one screen to weave ideas together. They indicated that the threaded interface frequently led to too many branched discussions that delineated from the main discussion topics and caused them to lose focus on the discussion, which often left them engaged in side conversations. 


\subsubsection{Sense of discussion context}

Although students valued flat-structured discussion boards, they admitted it was extremely difficult and confusing for them to relate to the context of the discussion. The sense of "context" can become lost quite easily, especially because context is not immediately obvious to who is responding to whom. One participant indicated, "It is easier to see who's "bustin" down on you, and easier to answer." One participant commented, "I have no idea if I am responding correctly or not... Where is everything?...I even couldn't find my own posting." Another participant echoed similar thoughts, "The discussion was chaotic...It's less muddy on DB [threaded]." One participant summarized well in sense of context, “... I was merrily responding to various postings thinking they would be connected directly to each post. After I went back see the fruits of my labor I was surprised to see most of my fruit seemed to be all on my very own tree."

\subsubsection{Discussion posting skills}

Flat-structured interface requires students to develop a different set of posting skills. Flatstructured interface is new to all participants while they are fairly familiar with threaded format. One participant expressed the frustration by "Not having any training on how to use the wiki there is great confusion... So out of frustration... some of us are responding by starting new threads." Some participants perceived posting skills should be "the best way for us to figure out the benefits is to use the wiki and play with it to try to and figure it out." Another participant echoed with "Like anything the best way to learn is through trial and error."

\subsubsection{Mental model}

A different mental model from threaded discussion is necessary to engage effectively with the flat-structured format, which left participants frustrated as one quoted, "I just dont [don't] understand how us taking a while to figure out ...it's a waste of our time." Another comment was made in that, "I have been trained to use threaded discussion...that is why myself (and others) had such difficulty becoming comfortable with that Web 2.0 tool." Participants are accustomed to threaded interface; therefore, they applied a threaded format mental model to participate in flatstructure discussions. Some participants were able to shift or become aware they needed to shift their mental models to participate in flat-structured discussions. One participant commented that "Web 2.0 tools support online learning by allowing students to organize their own learning content." Another participant offered valuable reflections:

I was a little frustrated with the discussion format in Wetpaint because I was not able to see who was responding to who's post. With vista, the discussions are threaded making it easy to follow. This is how I have been trained to read discussions. I was not aware of how to organize the discussions in Wetpaint, and after reading the site that was given to us, I go back once in awhile to play around with the "tags" and see what new things I can do.

\subsubsection{Learning Perceptions Shift}

Participants observed flat-structured discussions forced them to shift their learning to a new way. "I believe Web 2.0 and similar tools are great for discussion forums, chat rooms and the like," said one participant. Participants reflected that they should be ready for encouraging these learning disruptions, “... they best support online learning by forcing both teachers and students alike to think in new ways...CMS can become easily predictable and stagnant. The introduction and use of Web 2.0 
tools forces the lethargy out of those involved as it becomes necessary to view and learn a new way of learning."

\subsection{Social Dimension}

Context-oriented discussion environments and social networking features emerged from participants' discussion experiences. Participants manifested threaded discussions were more task-oriented and content-oriented while flat-structured discussions afforded more and richer social context on discussions and other participants due to social networking features. Online discussion, as one form of CMC, has been considered task-oriented (Culnan, \& Markus, 1987) and less social. One participant commented on threaded discussions, "I read in another post that it really doesn't take the time to get to know one another and just chat for the sake of chatting." Another participant emphasized the importance of social context, "We often learn by simply discussing each other's life experiences...it should be through the medium of school work that opens the doors for other types of learning to take place."

Unlike threaded discussion boards, flatstructured discussion boards are normally equipped with social networking features that allow users to upload their pictures or avatars, and manage their profiles to create and to enhance their online social identities. In this study, participants were required to create their profiles and upload their picture or avatar as part of the flat-structured discussion activities. Participants value social networking features within flat-structured discussions, “... incorporating features like profiles and personal pictures starts to meld the class realm with our real lives and the social realm." In fact, participants commented that pictures and profiles made the postings rendered by others more meaningful while reading discussion postings. Participants indicated,
"Personally, I learn better if I know my classmates better. In other classes, we do self introduction. That is good but I feel with the profile with pictures, I know them better and I think I have a better idea of what they are talking about.." Additionally, Wetpaint wiki is equipped with social networking widgets to enhance social bonding such as "Member Highlights," "Top Contributors," and "Who is talking." Participants expressed that these social network widgets helped them know their classmates better and allowed further understanding of what has been contributed by which community members.

Flat-structured discussions come with a social tagging feature to enhance discussions. With social tagging, participants indicated it infuses social dimension to enhance their understandings of their classmates' postings. One participant remarked, "Sometimes I had difficulty to comprehend someone's postings. With social tagging, it gives me better ideas (about) on the focus and themes of each posting because the author attached keywords to each posting." Social tagging was not required in this study; however, participants were encouraged to apply tags to their postings.

\subsubsection{Networking dimension}

Social network linkages play critical roles in flat-structured discussions while threaded discussions do not offer these features. The issues of social tagging, widgets, RSS, tag cloud, iGoogle, and $\log$ in were critical to discussion experiences of participants.

Because RSS and social tagging were encouraged to be applied to flat-structured discussion posting/discussion topic, students indicated that organizing RSS and providing tags engaged them in deeper thinking because they could determine how they would like their ideas to be presented. Threaded discussions 
were easier to follow, but flat-structured discussions required students to apply deeper cognitive thinking. Based on the observations, students made better and more comprehensive networks of references.

Several participants observed the value of social tagging in online discussions while participating in flat-structured discussions. These comments revealed that participants understood the values of social tagging even though they were still "confused" but observed the value of applying tagging to resolve the loss of discussion context. Participants stressed social tags as critical learning tools, "As students we can use Web 2.0 tools to stimulate learning amongst each other by tagging and adding information within the data source."

One participant made a particularly strong claim for social tagging:

I have been active in several online threaded communities and to me they seem very organized...in what sequential order. The tool...tags, and "was this useful" I have encountered in other areas...they are still new to me...but I am wishing that I had to continue Wetpaint so that I was forced to become more comfortable and familiar with...this emerging style.

Many participants were not accustomed to organizing their online discussions; therefore, RSS, tag clouds, wiki gadget/droplet on iGoogle, and other social network widgets (E-mail Notification, Recent Site Activity, Do you find this valuable? Most Recent Posting) were not utilized or applied effectively. Most participants did not understand what they were, how they worked, and how they might be integrated to support online discussions.
Managing multiple accounts to participate in discussions caused participants inconvenience and confusion. Flat-structured discussions on Wetpaint required participants to create a new account to participate in the discussions. Participants commented on utilizing multiple accounts "I don't have the personal memory capacity to remember all my passwords..." and preferred "one less thing to sign into."

\subsubsection{Integration Dimension}

The issues of collaboration effectiveness and community sense emerged from both discussion formats. Participants reflected that flat-structured discussion boards allowed them to engage in more collaborative tasks and generated a greater sense of community. Each posting was more "isolated" in threaded format because the reply posting was onelevel up from the parent posting. With various social networking features, participants felt their postings weaved together more effectively. This affords better collaborative and community learning. In the flat format, participants engaged in more effective collaboration, "...building a community and communicating between each other to learn more." Additionally, participants took pride being engaged in a new method of collaboration to build an online community, "... learn virtually anywhere and anytime. It can give some students pride in what they do by posting what they have learned."

\section{Discussion}

This study investigated the experiences of online graduate students in threaded and flatstructured discussion boards. Both formats critically enhanced and inhibited online discussions of students. Clearly, both formats empower discussion participants from different directions to engage them in a more meaningful, 
deeper, and higher order of thinking. Concluding that one format is better or more effective than the other or to argue that educators should seek the potential to apply one tool to replace the other is inappropriate. Each discussion format has its own strengths and weaknesses and this must be considered when using one format over the other. In fact, replacing the old one with the new one may result in ineffective and negative learning experiences and outcomes because both formats require learners to develop different mental models, posting skills, technical skills, and perceptions of learning values. The issue is not using one more or one less tool with which to learn, because learning can occur with either. Perhaps the more important questions to ask are: What are the more effective ways to engage learners in active interaction in online discussions? How can we engage learners in active learning in managing, organizing, and making more effective knowledge constructions in online discussion learning processes? What tools should be used and how should these tools be integrated to enhance online discussions?

The concepts of Open Network Learning Environment (ONLE) and personal learning environment (PLE) should be integrated to reach effective network discussions. This study proposes that learners should shift their roles from online learners to "network" learners, while instructors should shift their instruction paradigms from online discussions to "network" discussions. To reach interactive and effective network discussions, effective network discussion tools should provide multiple discussion interfaces such as threaded, nested, and flat integrate multiple social and networking features such as social tagging, tag or word clouds, RSS, widgets, profiles, and pictures or avatars. With multiple interfaces and multiple social network features, learners are required to engage in an active meta-cognitive and self-regulated learning processes by selecting and applying different and multiple interfaces and social network features to organize, to examine, to analyze, to comprehend, and to participate in network discussion knowledge interaction and sharing effectively to enhance participants' deeper, higher order, and critical thinking skills. Multi-dimensional network discussions would engage network learners in effective reading (text, auditory, \& visual), critical reflecting, displaying (information visualization) and doing (tactile, kinesthetic, and exploratory manipulating information). To engage learners in ONLE and PLE, allowing them to select and engage in multiple discussion interfaces and social network features is needed. Network learners define their own organizational scaffolding learning structures rather than follow pre-determined hierarchal structures.

Currently, some threaded and flatstructured discussion boards offer basic multiple discussions interfaces to support network discussions but without wide ranges of interfaces and social networking features. For example, Moodle's discussion boards provide multiple interfaces to allow learners to participate in discussion boards: flat, threaded, and nested. Other Web 2.0 discussion boards provide flat discussion boards with social and network features such as social tagging, RSS, widgets/gadgets, profiles, and pictures.

\section{Recommended Strategies}

Having effective network discussion tools does not result in completely effective learning environments. Effective instructional strategies should be developed and integrated into network discussion activities. Based on the results of this study, effective strategies are recommended to ensure that network learners participate in effective network discussions. 
In the area of cognitive dimension, the following instructional strategies are recommended:

- Provide warm-up exercises for the discussion board before any graded discussions take place.

- Explain the different values for both discussion formats.

- Explain that both discussion formats require different mental models to construct knowledge.

- Social tags should be encouraged or required.

- Integrate learner moderation to enhance a higher level of learning responsibility.

- Require learners to create, manage, and organize network discussions of their own through Personal Learning Environment (PLE) on a personal web portal, such as iGoogle, Pageflakes, or NetVibes.

In the area of social dimension, the following instructional strategies are recommended:

- Encourage or require students to create their own profile and share their pictures or avatars to enrich social context.

- Encourage learners to join social networks by requesting entry as friends, fans, or followers.

- Apply a third party social network tool to support social relationships among learners and instructors.

- Apply social network widgets, such as Top Contributors, Member Highlights etc. to tighten social bonding.

In the area of networking dimension, the following instructional strategies are recommended:

- Provide social tagging strategies.

- Require or encourage course members to subscribe to RSS discussion.

- Require or encourage learners to integrate RSS readers to organize discussion postings: such as Google Reader into iGoogle.

- Apply tag and/or word clouds as information visualization to support discussions.

- Provide tutorials for subscribing to RSS, social tagging, and other social \& networking mechanisms.

- Apply other Web 2.0 tools to organize discussion board postings, such as linking discussion board postings to personal blogs with widget, social bookmarking (Delicious), social networking site (Facebook), social annotation (Diigo), or PLE (iGoogle).

- Instructors select the tools with multiple formats (threaded, nested, flat, audio, video, \& mobile) and multiple social network features to create NLE to allow learners to manage and organize their PLE.

In the area of integration dimension, the following instructional strategies are recommended:

- Design and engage learners in network collaborative discussion activities.

- Integrate student group moderations into discussions to promote collaborative learning community.

- Encourage peer support by creating peer support discussion boards where students can post questions and allow students to respond.

\section{Future Research}

New network discussion learning instructions have emerged from this study. Educators and researchers should go beyond "online" discussion and continue examining the values, issues, effectiveness, and learning experiences of these new "network" discussion of instructional design. Below are a few 
suggested important topics to be examined to obtain a better understanding in supporting effective network discussions in terms of examining the how and what factors.

Regardless what discussion formats, threaded or flat-structured, the overall goals for online discussions do remain the same to engage learners in knowledge construction and to teach critical thinking skills. Future research should examine what and how flat-structured discussion format may impact learners' knowledge construction and their critical thinking skills. These examinations should not be limited to learners' perceptions, but also their actual gain of knowledge constructions and critical thinking skills.

Additionally, flat-structured discussion in network learning environments are frequently featured with social and network mechanisms such as social tagging, social networking, RSS feeds etc. Researchers should also investigate what and how these social and network mechanisms may impact learning outcomes in online discussion environments. Connectivism (Siemens, 2005) emphasized values of connecting people, resources, and tools in network learning environments and suggests that network discussions such as flat-structured discussion, should not be constrained to a single tool. To integrate multiple Web 2.0 tools to support network discussion should be examined whether it may impact online learning.

\section{Conclusions}

When a new technology is introduced to support learning, educators commonly find a way to replace old technology for the same function. Innovative network learning designs require educators to seek disruptive ways to integrate emerging network learning technologies. This requires educators to experience a fundamental change in pedagogical concepts and practices. Dede (2008) argued that effective strategies are to provide multiple specialized tools instead of a single instrument to complete all tasks. In innovative and disrupted learning environments, educators should build Open Network Learning Environments (ONLE) as interoperable learning infrastructures (Bush \& Mott, 2009) within learners and allow instructors to select appropriate, multiple tools to craft their Personal Learning Environments (PLE). PLE in ONLE is increasingly seen as a means for self-directed and collaborative learning, where individual learners construct their own agendas and learning organizations to satisfy their own learning goals (Sclater, 2008). With multiple tools and social network features, network discussion environments allow learners to construct and to contribute their knowledge by self-organized and selfmanaged discussion topics, conversations, and content (Mott, 2010). The researchers and educators should critically examine how they can empower network learners to define their organizational scaffolding network learning structures and environments rather than follow the pre-determined hierarchal structures defined by technologies.

\section{References}

Bonk, C., \& Dennen, V. (2007). Frameworks for design and instruction. In Handbook of distance education, In: M.G. Moore, Editor (2nd ed., pp. 233-246). Mahwah, NJ: Lawrence Erlbaum Associates.

Bush, M., \& Mott, J. (2009). The transformation of learning with technology learnercentricity, content and tool malleability, and network effects. Educational Technology, 49(2), 3-20. 
Cheong, C. M., \& Cheung, W. S. (2008). Online discussion and critical thinking skills: A case study in a Singapore secondary school. Australasian Journal of Educational Technology, 24(5), 556-573.

Culnan, M. J., \& Marcus, M. L. (1987). Information technologies. In In F.M. Jablin, L.L. Putnam, K.H. Robers, \& L.W. Porter (eds.), Handbook of organizational communication: An interdisciplinary perspective (pp. 420-443). Newbery Park,: CA: Sage.

Dede, C. (2008). Theoretical perspectives influencing the use of information technology in teaching and learning. In J. Voogt and G. Knezek, (Eds.), International Handbook of Information Technology in Primary and Secondary Education (pp. 4359). New York: Springer.

Educause. (2008). 2008 Horizon Report. Retrieved September 22, 2008, from http:// connect.educause.edu/Library/ELI/2008H orizonReport $/ 45926$ ?time $=1224635003$

Feldstein, M. (2005, March 30). Threaded discussion interfaces: A research challenge. e_literate. Retrieved July 16, 2009, from http://mfeldstein.com/threaded_discussion_ interfaces_a_research_challenge/

Garrison, D. R., Anderson, T., \& Archer, W. (2001). Critical inquiry in a text-based environment: Computer conferencing in higher education. The Internet and Higher Education, 2(2-3), 87-105.

Godwin-Jones, R. (2006). Tag clouds in the Blogosphere: Electronic literacy and social networking. Language Learning \& Technology, 10(2), 8-15.

Hillman, D. C.A., Willis, D. J., \& Gunawardena, C. N. (1994). Learner-interface interaction in distance education: An extension of contemporary models and strategies for practitioners. American Journal of Distance Education, 8(2), 30-42.

Lee, M. J. W., Miller, C., \& Newnham, L. (2008). RSS and Content Syndication in Higher
Education: Subscribing to a New Model of Teaching and Learning. Educational Media International, 45(4), 311-322.

de Leng, B. A., Dolmans, D. H. J. M., Jobsis, R., Muijtjens, A. M. M., \& van der Vleuten, C. P. M. (2009). Exploration of an e-learning model to foster critical thinking on basic science concepts during work placements. Computers \& Education, 53(1), 1-13.

Mott, J., \& Wiley, D. (2009). Open for learning: The CMS and the open learning network. In Education, 15(2). Retrieved July 16, 2009, from http://ineducation.ca/article/openlearning-cms-and-open-learning-network

Richardson, J. C., \& Ice, P. (2010). Investigating students' level of critical thinking across instructional strategies in online discussions. Internet and Higher Education, 13(1-2), 52-59.

Sclater, N. (2008). Web 2.0, personal learning environments, and the future of learning management systems. ECAR, 2008(13). Retrieved July 16, 2009, from http://www. educause.edu/ECAR/Web20PersonalLearn ingEnvironme/163047

Tu, C., Blocher, M., \& Roberts, G. (2008). Constructs for Web 2.0 learning environments: A theatrical metaphor. Educational Media International, 45(3), 253-268.

Vonderwell, S., Liang, X., \& Alderman, K. (2007). Asynchronous discussions and assessment in online learning. Journal of Research on Technology in Education, 39(3), 309-328. 


\section{Contact the Authors}

\section{Chih-Hsiung Tu, Ph.D.}

Northern Arizona University

E-mail: Chih.Tu@Nau.edu

Michael Blocher, Ph.D.

Northern Arizona University

E-mail: Michael.Blocher@Nau.edu

Lawrence Gallagher, Ed.D.

Northern Arizona University

E-mail: Lawrence.Gallagher@Nau.Edu 